\title{
Vertical bone augmentation utilizing a titanium-reinforced PTFE mesh: A multi-variate analysis of influencing factors
}

\author{
Istvan A. Urban ${ }^{1,2,3,4}$ (D) | Muhammad H. A. Saleh ${ }^{2,5}$ | Andrea Ravidà2 (D) | \\ Andras Forster $^{4}$ | Hom-Lay Wang ${ }^{2}$ (D) | Zoltan Barath ${ }^{6}$
}

${ }^{1}$ Graduate Implant Dentistry, Loma Linda University, Loma Linda, CA, USA

${ }^{2}$ Department of Periodontics and Oral Medicine, University of Michigan School of Dentistry, Ann Arbor, MI, USA

${ }^{3}$ Departement of Periodontics, University of Szeged, Szeged, Hungary

${ }^{4}$ Urban Regeneration Institute, Budapest, Hungary

${ }^{5}$ Department of Periodontics, University of Louisville School of Dentistry, Louisville, KY, USA

${ }^{6}$ Department of Prosthetic Dentistry, University of Szeged, Szeged, Hungary

\section{Correspondence}

Hom-Lay Wang, Department of Periodontics and Oral Medicine, University of Michigan School of Dentistry 1011 North University Avenue, Ann Arbor, MI 48109-1078, USA.

Email: homlay@umich.edu

\begin{abstract}
Objective: To clinically evaluate the use of a titanium-reinforced PTFE mesh for vertical bone augmentation (VBA) of deficient alveolar ridges.

Materials and methods: This case series documented consecutive patients treated for VBA with a newly developed PTFE mesh. VBA was performed in anterior and posterior, maxillary and mandibular arches using anorganic bovine bone combined with autogenous graft in a 1:1 ratio. Healing time from initial surgery to re-opening was recorded. Baseline vertical deficiency, absolute bone gain (gross height gained), and relative gain (percentage of defect fill with respect to the baseline deficiency) were registered.

Results: Fifty-seven patients ( 65 defects) were included in the analysis. The mean baseline vertical deficiency was $5.5 \pm 2.6 \mathrm{~mm}$. The mean absolute bone gain was $5.2 \pm 2.4 \mathrm{~mm}$. A relative gain of $96.5 \pm 13.9 \%$ was achieved. Overall, $89.2 \%$ of cases showed complete regeneration, which occurred in all sites with baseline deficiencies of $<5 \mathrm{~mm}$, in $95.6 \%$ of sites with $5-8 \mathrm{~mm}$ deficiencies, and in $89.4 \%$ of sites with $>8 \mathrm{~mm}$ deficiencies. Each 1-mm addition to the baseline height deficiency increased the likelihood of incomplete bone regeneration by 2.5 times. Defect location had a statistically significant but a limited clinical impact on the bone height gained $(<0.5 \mathrm{~mm})$. Complications were observed in three cases (3\%).

Conclusions: Vertical bone augmentation with titanium-reinforced PTFE mesh and a mixture of autologous bone and xenograft is a safe and predictable procedure. The extent of the baseline vertical deficiency influences the percentage of bone gained.
\end{abstract}

\section{KEYWORDS}

biomaterials, bone regeneration, bone substitutes, guided tissue regeneration, surgical techniques

\section{1 | INTRODUCTION}

Long-term implant success occurs only when implants are placed in biologically and prosthetically favorable positions; alveolar ridge deficiencies impede meeting these requirements (Buser et al., 2017; Mailoa et al., 2017). Extensive bone and soft tissue defects result from periodontal disease, trauma, or other pathologies (Hammerle \& Tarnow, 2018). While block grafting had 
been considered the optimal treatment to correct hard tissue deficiencies, guided bone regeneration (GBR) was found to generate results on par with block grafting (Milinkovic \& Cordaro, 2014). A systematic review and meta-analysis on vertical bone augmentation (VBA) concluded that GBR using barrier membranes produced comparable bone gain and fewer postoperative complications compared with block grafting (Urban et al., 2019). Though, other reviews have demonstrated the superior results for block grafting (Chiapasco \& Casentini, 2018) and higher complication rates for GBR (Chiapasco \& Zaniboni, 2009).

Autologous bone remains the gold standard material for grafting due to its osteogenic, osteoinductive, and osteoconductive characteristics but is quantitatively limited, resorbs faster, and frequently requires a second donor site relative to other graft types (Cordaro et al., 2002; Papageorgiou et al., 2016; Sbordone et al., 2009). Supplementing particulate autogenous bone with anorganic bovine bone-derived mineral (ABBM) significantly slows the rate of graft resorption and requires less autogenous harvesting (Maiorana et al., 2005; Wen et al., 2018). Combining graft types is an efficient approach for ridge regeneration.

Guided bone regeneration uses barrier membranes to create space above the bony defect and subperiosteally and to exclude faster-migrating connective tissue and epithelial cells, giving osteoprogenitor cells the space and time necessary to colonize and fill with bone the surgically constructed area (Polimeni et al., 2006). Occlusive membranes, however, may decelerate angiogenesis, as vessels from the subperiosteum are blocked by the barrier, permitting revascularization mainly from the residual ridge (Wang \& Boyapati, 2006). VBA is particularly demanding biologically since osteogenesis and angiogenesis rely only on basal bone, outside any bony envelope.

Studies have demonstrated predictable results with GBR using non-resorbable membranes that remain unexposed (Machtei, 2001; Nowzari \& Slots, 1995). Non-resorbable membranes mandate removal, which is a non-issue when pre-implantation GBR is planned but a drawback when GBR simultaneous to implant placement occurs. Absorbable membranes do not necessitate removal and are clinically effective and cell compatible; as such, they are preferred by many clinicians (Jung et al., 2013; Rothamel et al., 2004). However, some systematic reviews have reported that absorbable membranes are more complication-prone than non-resorbable ones, particularly in terms of membrane exposure (Elnayef et al., 2018; Lim et al., 2018; Urban et al., 2019): The major concern with absorbable membranes is that they maintain space less well than non-absorbable barriers because they lack form stability, which leads to graft migration, membrane collapse, and insufficient bone formation (McGinnis et al., 1998). A systematic review has also shown that the success rates of implants placed in ridges regenerated with titanium mesh were comparable to those of implants placed in native bone, and of implants placed in bone regenerated with either absorbable or non-resorbable membranes (Ricci et al., 2013). Long-term follow-up for implants after VBA using titanium mesh also demonstrated high survival rates (Roccuzzo et al., 2017).
Our group has shown that using titanium-reinforced dPTFE membranes with a mixture of autologous bone and xenograft produce satisfactory VBA outcomes (Urban et al., 2014, 2015). A hybrid structure combining classic titanium mesh and PTFE membrane features has been introduced (RPM ${ }^{\mathrm{T}}$-Reinforced PTFE Mesh, Cytoplast ${ }^{\circledR}$; Osteogenic Biomedical). This PTFE mesh is composed of circular macropores that allow direct communication between the surgically created space and the overlying periosteum, allowing the area to be more readily revascularized and populated by progenitor cells per the manufacturer. The titanium frame of this structure maintains the space essential for VBA.

Regardless of the surgical approach, VBA may achieve approximately $4 \mathrm{~mm}$ of vertical bone gain (Elnayef et al., 2018; Milinkovic \& Cordaro, 2014; Urban et al., 2019). To date, we are not aware of any patient-based investigation on VBA using this hybrid PTFE mesh $\left(R_{P M}{ }^{\mathrm{TM}}\right)$. Our retrospective case series aims to clinically evaluate using a titanium-reinforced PTFE mesh to correct vertical ridge deficiencies prior to implant placement and to assess factors influencing treatment outcomes.

\section{1 | Primary study objectives}

1. To evaluate the amount of bone height gain in millimeters and as a percentage of the baseline deficiency from VBA using a titanium-reinforced PTFE mesh with mixed autogenous and ABBM particulate grafts.

2. To examine the influence of defect location, baseline deficiency extent, and membrane exposure on augmentation height.

3. To report the incidence of surgical and post-surgical complications associated with this treatment.

\section{MATERIAL AND METHODS}

Fifty-seven consecutive patients who had been treated using titanium-reinforced PTFE mesh with mixed autogenous and ABBM particulate grafts for pre-implantation VBA between August 2016 and June 2019 were included for analysis. All patients were treated in one private practice (Urban Regeneration Institute, Budapest, Hungary). All VBA procedures were performed by the same experienced practitioner (IU). Implant placement and subsequent prosthetic treatments were performed by author IU or other private practitioners. This study was approved by the Institutional Review Board for Human Studies with the following code (118/2020SZTE). Strengthening the Reporting of Observational Studies in Epidemiology (STROBE) guidelines were followed during the preparation of the manuscript.

\section{1 | Inclusion and exclusion criteria}

Included patients had vertical bone insufficiency that precluded stable dental implant placement or that would result in poor 
crown-to-implant ratios or esthetic compromise. Participants were required to have good physical health and good oral hygiene prior to treatment (plaque index of <10\%) (Silness \& Loe, 1964).

Patients were not recruited if any of the following criteria were met:

1. Bone augmentation procedures not using titanium-reinforced PTFE mesh.

2. Heavy smokers ( $>10$ cigarettes/day).

3. History of local radiation therapy within the previous 5 years.

4. Uncontrolled diabetes mellitus.

5. Alcoholism or chronic drug abuse.

6. Any uncontrolled medical conditions.

\subsection{Surgical procedure}

Potential risks and benefits of VBA procedure were reviewed presurgically with all enrolled patients. Written consent was obtained from all patients prior to surgery. All participants received prophylactic systemic antibiotic coverage with 500 mg amoxicillin tid or, in the case of penicillin allergy, $150 \mathrm{mg}$ clindamycin qid $24 \mathrm{hr}$ prior to surgery.

A mid-crestal incision was made in the keratinized mucosa of the edentulous site to be augmented, and sulcular incisions were made around adjacent teeth. Periosteal elevators were used to raise full-thickness mucoperiosteal flaps extending at least $5 \mathrm{~mm}$ apical to the alveolar crest; particular care was given to sites with thin mucosa or minimal to no keratinized tissue to avoid flap perforation. To enhance access, two vertical releasing incisions were made at least one tooth away from the surgical site. The depth and location of the vertical releasing incisions, as well as the technique used for flap management, depended on the depth of the vestibule and the extent of the existing defect (Urban et al., 2016). For mandibular cases, lingual flaps were elevated to the mylohyoid muscle attachment, which was then bluntly separated (Urban et al., 2017).

Each recipient site was decorticated using a small round bur to increase blood supply to the recipient bed. A particulate autograft was harvested with a bone scraper from intraoral sites adjacent to the defect (Osteogenic Biomedical). The amount of bone harvested was based on the amount of graft needed. Autogenous bone particulate was mixed with ABBM (Bio-Oss, Geistlich Pharma, AG) in a 1:1 ratio and positioned on the residual ridge to mimic the desired bony morphology.

The area to be covered by mesh was estimated using University of North Carolina-15 probe (UNC-15), and an appropriately sized titanium-reinforced PTFE mesh $\left(\mathrm{RPM}^{\mathrm{T}}\right)^{\mathrm{n}}$ ) was selected, trimmed, and placed to completely cover the graft and at least $2 \mathrm{~mm}$ of adjacent native bone. The mesh was stabilized on the lingual/palatal sides using titanium pins (Master Pin, Meisinger) or screws (Profix ${ }^{\circledR}$, Osteogenics Biomedical) (Urban et al., 2016). The PTFE mesh was covered with a native collagen membrane (Bio-Gide, Geistlich Pharma, AG) without stabilization.

Periosteal releasing incisions were carefully made to advance buccal flaps. In premolar sites, the mental nerve was protected, particularly cases with severe atrophy that demanded more apically extending vertical incisions. Lingual flaps were advanced based on the location of the mylohyoid muscle attachment and were handled according to three previously described zones of interest (Urban et al., 2017). Double-layered suturing was employed to create intimate tissue adaptation and prevent membrane exposure. In this technique, horizontal mattress sutures (GORE-TEX ${ }^{\circledR}$ CV-5 Suture, W.L. Gore \& Associates) were placed 4-5 mm from the incision line. Then, single interrupted sutures were used to secure all flap edges. The sutures were left undisturbed for at least 2-3 weeks. The surgical site was allowed to heal for at least 6 months. At re-entry, limited mucoperiosteal flaps were elevated to remove the PTFE mesh and titanium pins and/or screws and to perform implant placement. Figure 1 details the surgical procedure.

\section{3 | Post-surgical procedures}

A postoperative regimen of amoxicillin $500 \mathrm{mg}$ tid for 7 days or, in the case of penicillin allergy, $150 \mathrm{mg}$ clindamycin qid for 6 days was prescribed. A nonsteroidal anti-inflammatory drug (50 mg diclofenac potassium tid or ibuprofen $200 \mathrm{mg}$ tid) was prescribed for 1 week following the surgery. All patients were evaluated at 1, 2 or 3, and 4 weeks following surgery. Intra-operative and postoperative complications such as membrane exposure, intra-operative bleeding, and graft infection were recorded.

\section{4 | Data collection}

Patient information including gender, age at the time of the surgical treatment, and self-reported cigarette consumption was recorded. Intraoperatively, the extent of baseline vertical deficiency was measured by one provider (I.U) in millimeters from the residual ridge crest to one reference line using a UNC-15 probe. One of two reference lines was used to ensure consistent vertical measurements and to serve as ideal heights: (a) an imaginary line connecting the interproximal bone height between adjacent teeth; or (b) in the case of distal edentulism, an imaginary line connecting the proximal tooth bone height to the projected nonresorbed alveolar crest of the edentulous area. The vertical bone gain was evaluated at the time of implant placement (re-entry) and measured in the same fashion as described above. The horizontal distance between the site of measurement and the root surface/ non-resorbed alveolar crest of the nearest tooth was recorded to guarantee reproducible measurement in mesio-distal direction. 

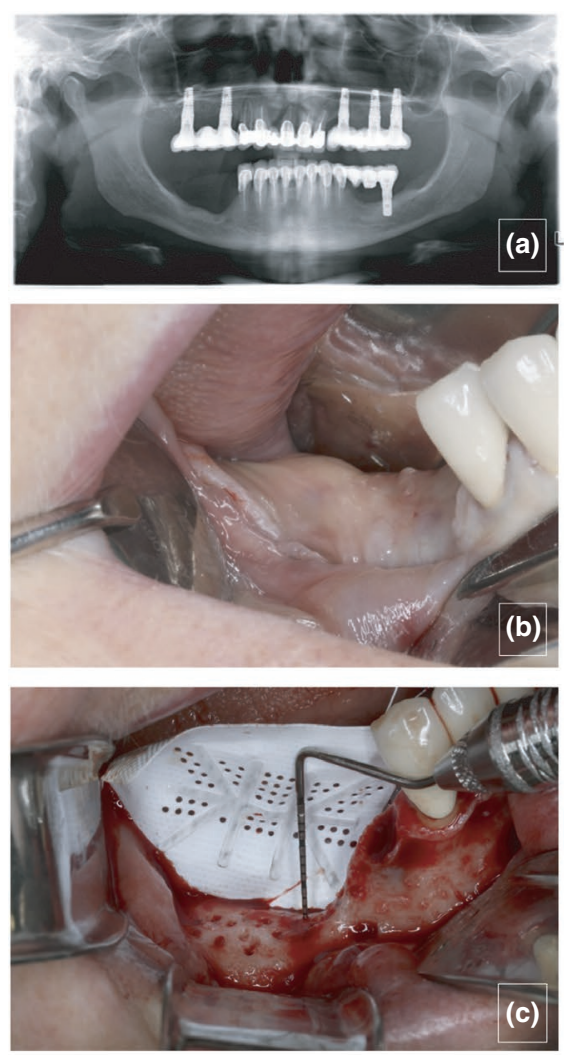
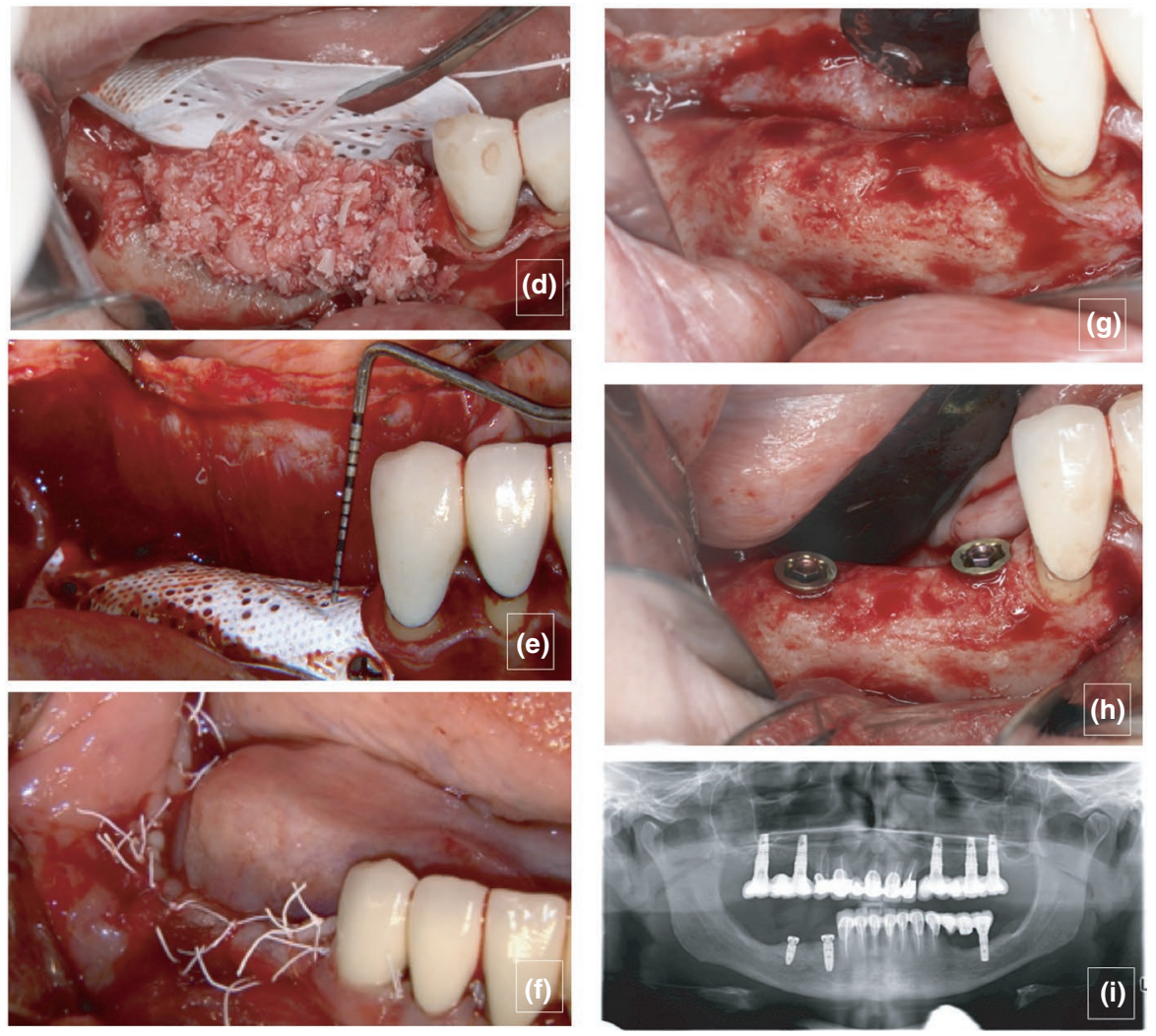

FIGURE 1 Representative case from the current cohort with a step-by-step demonstration of the surgical procedure. (a) Panoramic radiograph showing a posterior mandibular area that required vertical GBR. The mental nerve approximated the residual ridge crest. (b) Buccal view showing the atrophic ridge preoperatively. (c) Titanium-reinforced PFTE mesh was fixed in place after decortication of recipient site; the size of the vertical defect was $\geq 12 \mathrm{~mm}$. (d) Placement of particulate anorganic bovine bone combined with autogenous graft (1:1 ratio). (e) Titanium-reinforced PFTE mesh fixed in place with titanium pins and screws. A coronal flap advancement of nearly 15 mm was obtained before closure. (f) Tension-free flap closure was performed using the double-layer suturing technique. (g) Clinical view of the regenerated bone. Note the excellent dimensions and vital-looking ridge. (h) Two implants in place in biologically and prosthetically ideal positions. (i) Postoperative panoramic radiograph demonstrating implant position after successful VBA

Absolute gain was defined as the amount of bone gained in millimeters regardless of baseline vertical deficiency. Relative gain was defined as the percentage of the vertical deficiency that was resolved relative to the ideal height.

\section{5 | Statistical analysis}

Logistic regression using generalized estimation equations (GEE) was conducted to assess vertical bone gain differences and the probability of complete relative bone gain according to positional variables. Models were adjusted for defect size, healing time, age, and smoking; beta coefficients, odds ratio, and 95\% confidence intervals (via Wald chi-square test) were calculated. The significance level was set as $5 \%(\alpha=.05)$.

A post hoc power analysis determined that a sample size of 65 independent sites provided $85.1 \%$ power with $95 \%$ confidence for detecting mean gains of 4.5 and $6.0 \mathrm{~mm}$ as significantly different using linear regression. However, as not all sites were independent, a power correction was necessary. Each patient provided a mean of 1.14 sites, and a high within-subject correlation $(\mathrm{CCl}=0.9)$ was assumed, leading to a correcting coefficient of $D=1.13$. Therefore, 65 dependent sites provided the same power as 58 independent ones (80.1\%).

\section{RESULTS}

\section{1 | Patient characteristics}

The sample included 57 patients (65 defects) who underwent VBA using titanium-reinforced PTFE mesh. Twenty-one males (36.8\%) and 36 females (63.2\%) with a mean age of $51.9 \pm 11.8$ years (range: 28-78 years) were included. Each patient received surgery in one (86\%) or two different sites (14\%). $96.5 \%$ of the patients were nonsmokers; two patients were smokers. Demographic, clinical, and defect distribution characteristics are shown in Table 1; defect distribution is further described in Figure 2. 
TABLE 1 Demographic and clinical characteristics of the studied cohort. Number of patients (\%) or sites (\%), mean \pm standard deviation

\begin{tabular}{|c|c|}
\hline & $N(\%) /$ mean $\pm S D$ \\
\hline \multicolumn{2}{|l|}{ Patient level } \\
\hline$N$ patients & 57 \\
\hline Age (years) & $51.9 \pm 11.8$ \\
\hline \multicolumn{2}{|l|}{ Gender } \\
\hline Male & $21(36.8)$ \\
\hline Female & $36(63.2)$ \\
\hline \multicolumn{2}{|l|}{ Smoking } \\
\hline No & $55(96.5)$ \\
\hline Yes & $2(3.5)$ \\
\hline \multicolumn{2}{|l|}{$N$ sites on surgery } \\
\hline One & $49(86.0)$ \\
\hline Two & $8(14.0)$ \\
\hline \multicolumn{2}{|l|}{ Site level } \\
\hline$N$ sites & 65 \\
\hline \multicolumn{2}{|l|}{ Site } \\
\hline MAX anterior & $12(18.5)$ \\
\hline MAX right posterior & $11(16.9)$ \\
\hline MAX left posterior & $6(9.2)$ \\
\hline MAN anterior & $4(6.2)$ \\
\hline MAN right posterior & $15(23.1)$ \\
\hline MAN left posterior & $17(26.2)$ \\
\hline \multicolumn{2}{|l|}{$\mathrm{N}$ implants } \\
\hline 0 & $3(4.6)$ \\
\hline 1 & $7(10.8)$ \\
\hline 2 & 34 (52.3) \\
\hline 3 & $20(30.8)$ \\
\hline 4 & $1(1.5)$ \\
\hline Healing time (months) & $9.7 \pm 3.3$ \\
\hline \multicolumn{2}{|l|}{ Complications } \\
\hline No & $63(97)$ \\
\hline Exposure & $1(1.5)$ \\
\hline Infection & $1(1.5)$ \\
\hline \multicolumn{2}{|l|}{ Defect type } \\
\hline Vertical & $65(100)$ \\
\hline Defect size (mm) & $5.5 \pm 2.6$ \\
\hline$N$ of missing teeth & $3.2 \pm 1.2$ \\
\hline Maxilla & $3.1 \pm 1.5$ \\
\hline Mandible & $3.2 \pm 0.9$ \\
\hline
\end{tabular}

\section{2 | Bone gain analysis}

Table 2 and Figure 3a,b document ridge height changes post-VBA. The mean baseline vertical deficiency was $5.5 \pm 2.6 \mathrm{~mm}$. The absolute gain in vertical dimension from VBA was $5.2 \pm 2.4 \mathrm{~mm}$, corresponding a

\section{Distribution of defects (\%) by site}
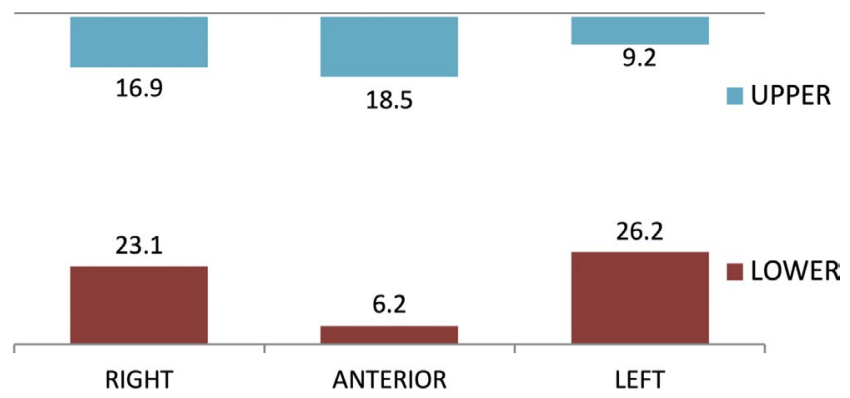

FIGURE 2 Distribution of the defect (\%) by site

\section{Defect size and absolute and relative gain}

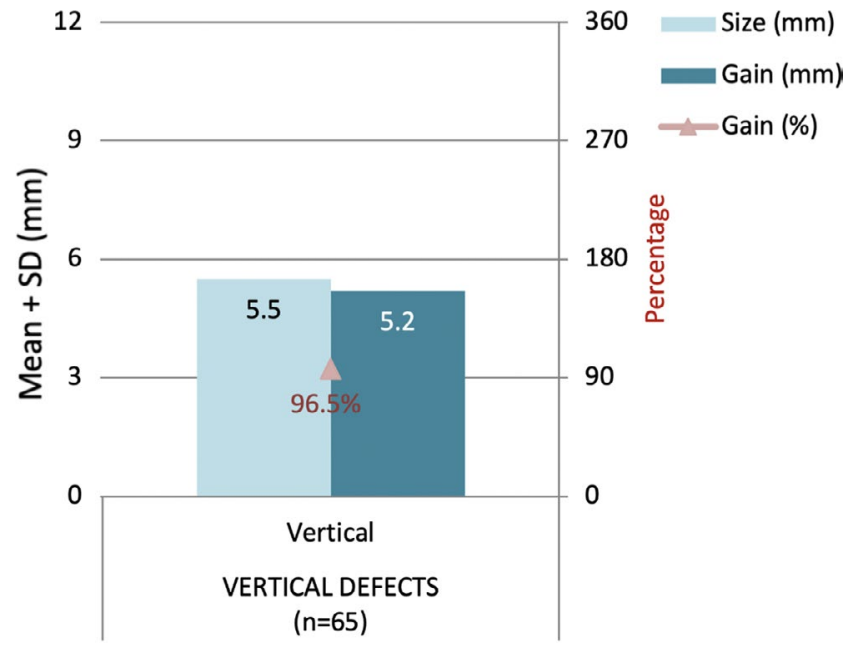

FIGURE 3 Absolute vertical gain $(\mathrm{mm})$ and relative vertical gain (\%) after vertical bone augmentation

mean relative height gain of $96.5 \pm 13.9 \% ; 89.2 \%$ of the sites presented complete regeneration, that is, elimination of vertical deficiency.

\section{3 | Influence of baseline vertical deficiency on absolute and relative bone gain}

Six defects out of 65 were not completely regenerated. Five of these sites had baseline vertical deficiencies of $\geq 10 \mathrm{~mm}$ (Figure $4 \mathrm{a}$ ), and one site with a $6 \mathrm{~mm}$ baseline deficiency was infected postoperatively. The probability of having complete regeneration was inversely proportional to defect size $(p=.005)$. Each 1-mm addition to baseline height deficiency increased the likelihood of incomplete bone regeneration by 2.5 times. Having a baseline deficiency of $5-8 \mathrm{~mm}$ reduced the relative gain by $6 \%$ compared with having a baseline deficiency $<5 \mathrm{~mm}(p=.045)$. Having a baseline deficiency of $>8 \mathrm{~mm}$ reduced the relative bone gain by $12 \%$ compared with having a baseline deficiency $<5 \mathrm{~mm}(p<.001)$ (Figure 4b). Multiple linear regression controlling for defect size, arch, healing time, and age (Table 3) 
TABLE 2 Changes of the dimensional parameters of the defects after bone augmentation

\begin{tabular}{|lllll|} 
Defect type & $\begin{array}{l}\text { Defect } \\
\text { size }\end{array}$ & $\begin{array}{l}\text { Absolute } \\
\text { gain }(\mathrm{mm})\end{array}$ & $\begin{array}{l}\text { Relative gain } \\
(\%)\end{array}$ & $\begin{array}{l}\text { Percentage of cases with } \\
\text { complete regeneration (\%) }\end{array}$ \\
\hline Vertical & $5.5 \pm 2.6$ & $5.2 \pm 2.4$ & $96.5 \pm 13.9$ & 89.2 \\
\hline
\end{tabular}

TAB LE 3 Multiple linear regression analysis on the relative vertical gain (\%) controlling for defect size, arch, healing time, and age

\begin{tabular}{lccc|} 
& $\begin{array}{c}\text { Regression } \\
\text { coefficient }\end{array}$ & $95 \% \mathrm{Cl}$ & $p$-value \\
\hline $\begin{array}{l}\text { Defect size } \\
<5 \mathrm{~mm} \text { (ref.) }\end{array}$ & 0.00 & & $<.001^{* * *}$ \\
$5-8 \mathrm{~mm}$ & -5.97 & -11.8 to 0.13 & $.045^{*}$ \\
$>8 \mathrm{~mm}$ & -11.9 & -17.8 to 5.89 & $<.001^{* * *}$ \\
\hline Arch & & & \\
Maxilla (ref.) & 0.00 & & \\
Mandible & -2.98 & -8.01 to 2.05 & .246 \\
Healing time & 1.34 & 0.08 to 2.60 & $.037^{*}$ \\
Age & 0.08 & -0.05 to 0.21 & .207 \\
\hline
\end{tabular}

${ }^{*} p<.05 ;{ }^{* * *} p<.001$.

identified healing time to be a significantly affected vertical growth ( $p=.037)$. Each 1-month addition to healing time increased relative gain by $1.34 \%$.

\section{4 | Influence of defect location on absolute bone gain: maxilla versus mandible}

There were 29 maxillary and 36 mandibular defects treated in this study. The baseline mean vertical deficiency was $5.3 \pm 2.5 \mathrm{~mm}$ in the maxilla and $5.6 \pm 2.7 \mathrm{~mm}$ in the mandible; there was no difference in this variable between the arches per GEE ( $p=.664)$. Mean absolute vertical bone gain was $5.1 \pm 2.2 \mathrm{~mm}$ in the maxilla and $5.3 \pm 2.6 \mathrm{~mm}$ in the mandible; there was no difference in this variable between the arches per multiple linear regression $(p=.596)$ (Table 4). Defect size $(p<.01)$ and healing time $(p<.05)$ significantly affected vertical gain. Smoking was not statistically relevant $(p=.220)$, but a large effect size $(\beta=-1.60)$ was observed.

\subsection{Influence of defect location on absolute bone gain: anterior versus posterior}

Out of the 29 maxillary vertical defects, 12 were anterior and 17 were posterior. The mean baseline vertical deficiency was $5.7 \pm 2.7 \mathrm{~mm}$ anteriorly and $5.1 \pm 2.4 \mathrm{~mm}$ posteriorly; these values were not statistically different $(p=.489)$. The mean absolute vertical gain was statistically higher in posterior sites than anterior ones by $0.36 \mathrm{~mm}$ ( $p=.048$ ) (Table 4). The extent of the baseline vertical deficiency $(p<.01)$ and smoking $(p<.05)$ significantly affected maxillary absolute gain (Table 4).

Out of 36 vertical defects, 4 were anterior and 32 were posterior. The mean vertical deficiency was $5.3 \pm 1.0 \mathrm{~mm}$ anteriorly and $5.6 \pm 2.9$ posteriorly; these values were not statistically different $(p=.540)$. The mean absolute vertical bone gain was significantly greater in anterior sites than posterior ones by $0.32 \mathrm{~mm}(p=.021)$ (Table 4). The extent of the baseline vertical deficiency and smoking significantly affected mandibular absolute gain.

\subsection{Influence of defect location on absolute and relative bone gain: anterior versus left posterior versus right posterior}

In the maxilla, there were 12 anterior, 11 right posterior, and 6 left posterior defects. The mean vertical deficiency was $5.5 \pm 2.9 \mathrm{~mm}$ on the right posterior side and $4.3 \pm 0.8$ on the left posterior side. There were no differences in absolute bone gain between maxillary left and right posterior sides per multiple linear regression $(p=.726)$. Maxillary anterior defects showed less bone gain compared with left and right posterior defects ( $p=.05$ ) (Table 4, Figure 5a).

In the mandible, there were 4 anterior, 15 right posterior, and 17 left posterior defects. The mean vertical defect size was $5.8 \pm 3.2 \mathrm{~mm}$ on the right posterior side and $5.5 \pm 2.6$ on the left posterior side, with no significant differences between the sides $(p=.72)$. A statistically significant difference in vertical bone gain was detected between the mandibular anterior, left posterior, and right posterior areas ( $p=.028$ ) (Table 4 and Figure $5 b)$. The relative vertical gain was $\mathbf{9 8 . 3 \%}$ for mandibular left posterior sites and $90.9 \%$ for right posterior sites ( $0.3 \mathrm{~mm}$ of absolute gain difference).

\section{7 | Post-surgical complications}

There were only two cases (3\%) with complications. One site had membrane exposure (at 1 week postoperatively); graft material became infected at a second site. For the first case, the exposed membrane was maintained for 2 months, and then, it was removed. For the second case, the infected membrane and graft were explored and removed after 10 days of healing.

\section{4 | DISCUSSION}

Vertical ridge augmentation using space-making frameworks and graft materials offers an ideal balance between the expected amount of 


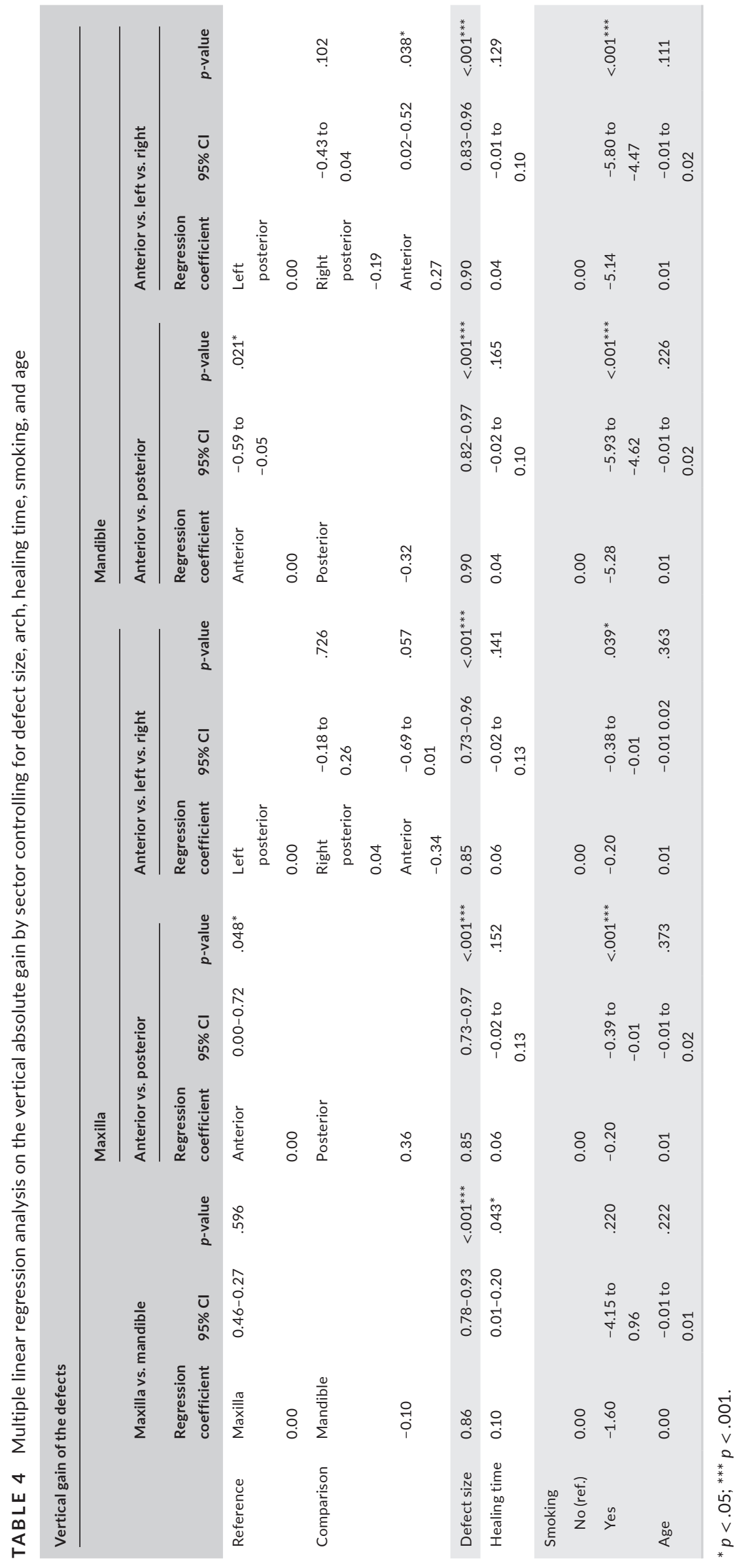




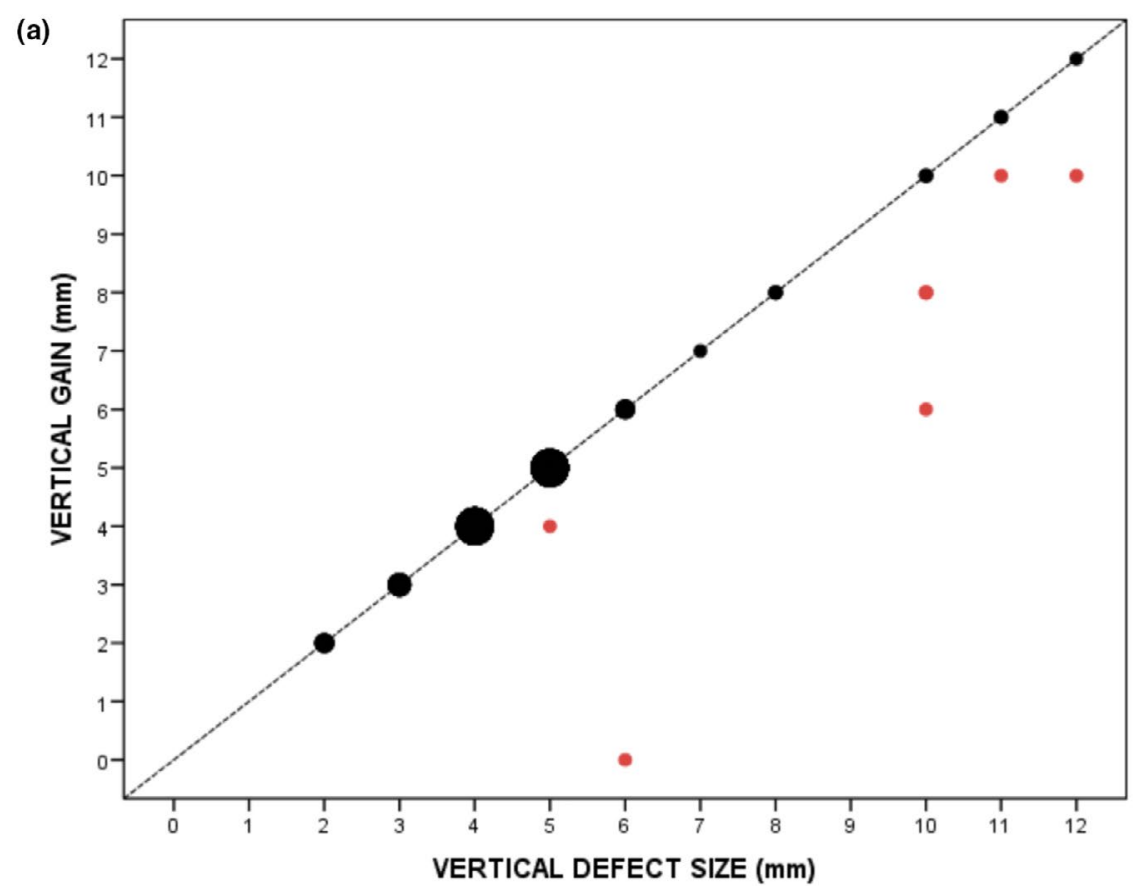

(b)

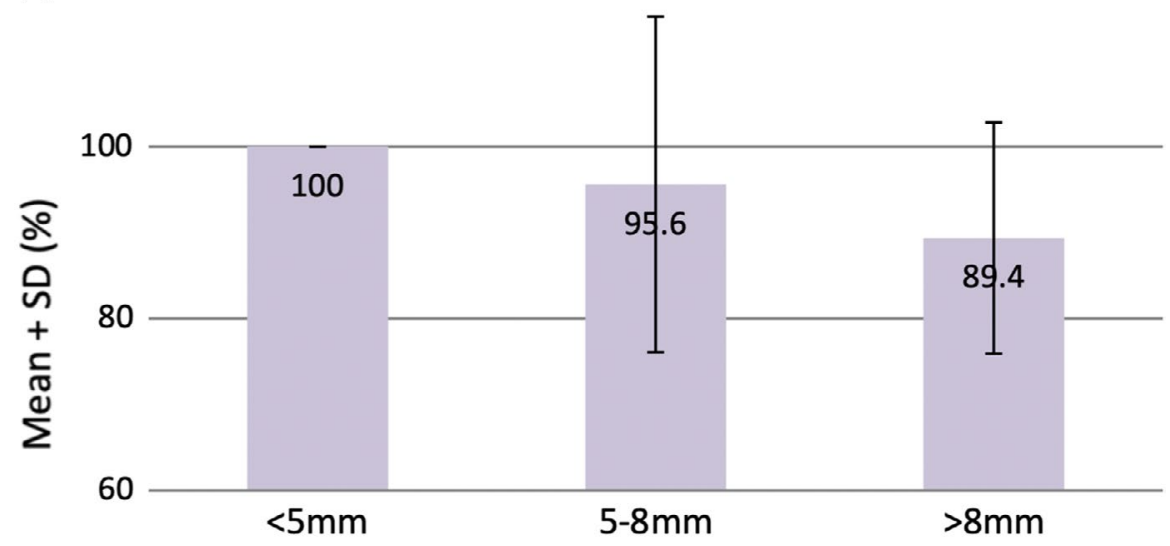

FIGURE 4 (a) Correlation between defect size and vertical gain. Red points represent cases that were not completely regenerated. Five such cases had baseline vertical deficiencies measuring $\geq 10 \mathrm{~mm}$. One defect with a baseline deficiency of $6 \mathrm{~mm}$ showed null gain. (b) Relative vertical gain according to baseline deficiency bone gain and postoperative complications compared with other interventions (Milinkovic \& Cordaro, 2014; Urban et al., 2019). The rationale of GBR is based on the creation of a sheltered, soft tissue cell-excluding area to promote osteoblast migration (Melcher, 1976). Dimensionally stable structures such as titanium-reinforced non-resorbable membranes or non-occlusive titanium meshes support vertical dimensions more reliably than cell-occlusive but pliant absorbable membranes. Dense polytetrafluoroethylene (dPTFE) is a commonly used nonresorbable barrier material, but it may exhibit subpar tissue adhesive properties that risk flap integrity (Park et al., 2009). Innovators have developed materials that better approximate the periosteum to the underlying graft. Our study utilized a titanium-reinforced PTFE mesh perforated by macropores that theoretically improves vascularization by permitting direct contact between periosteum and bone grafts. To our knowledge, this is the first human case series to record use of titanium-reinforced PTFE mesh for VBA.

\subsection{Agreement with previous studies}

Our case series achieved an absolute vertical bone gain of $5.2 \pm 2.4 \mathrm{~mm}$, which matched results reported in two previous case series by author IU using titanium-reinforced dPTFE membranes with the same bone graft composition that achieved mean height gains of 5.45 and $5.83 \mathrm{~mm}$ (Urban et al., 2014, 2015). In the current study, we found a relative bone gain of $96.5 \pm 13.9 \%$ and complete vertical ridge regeneration in $89.2 \%$ of cases. Compared with results from studies by the same author using nonporous dPTFE, titaniumreinforced PTFE mesh seems to provide similar or better vertical bone gain (Urban et al., 2014, 2015, 2016).

Other studies using titanium-reinforced expanded polytetrafluoroethylene membranes (ePTFE) reported similar results to ours. Urban et al attained a mean height gain of $5.5 \mathrm{~mm}$ using titaniumreinforced ePTFE membrane and autologous bone for maxillary and 
FIGURE 5 Absolute and relative vertical gain in the (a) maxilla and (b) mandible

\section{(a) Absolute and relative vertical gain in the maxilla}

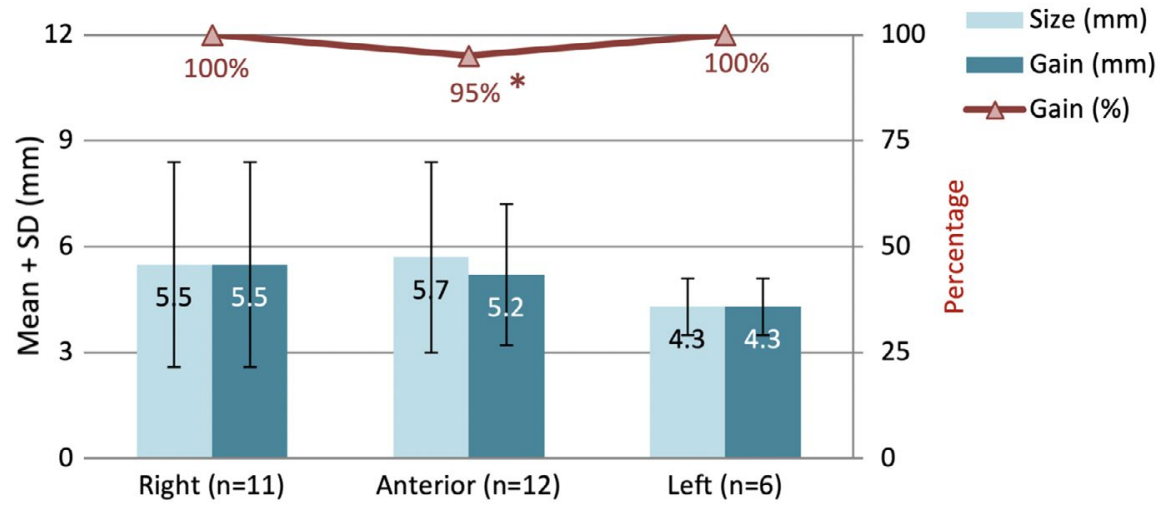

(b) Absolute and relative vertical gain in the mandible
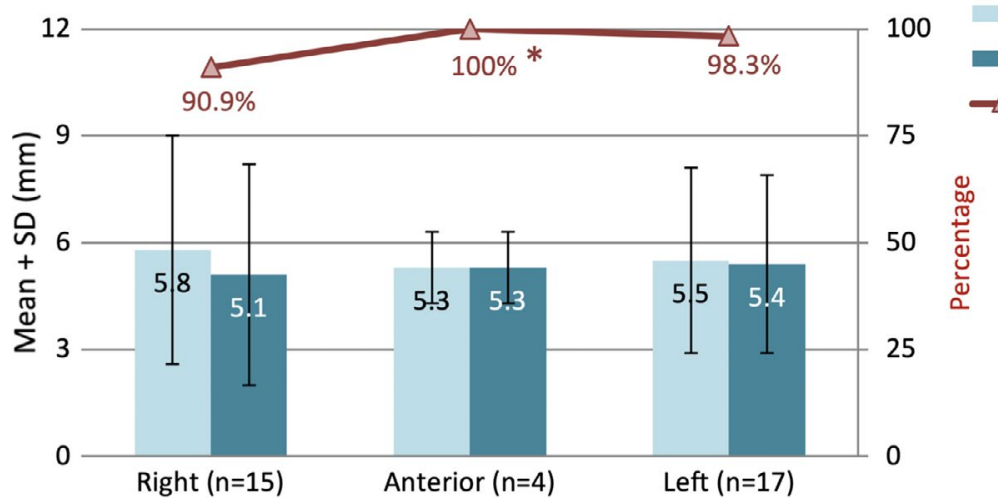

mandibular, anterior and posterior VBA (staged or simultaneous) (Urban et al., 2009). Canullo \& Sisti demonstrated a mean height gain of $5.85 \mathrm{~mm}$ using titanium-reinforced ePTFE membranes with magnesium-enriched hydroxyapatite for maxillary and mandibular, anterior and posterior VBA (Canullo \& Sisti, 2010). Todisco achieved a mean vertical height of $5.3 \mathrm{~mm}$ using titanium-reinforced ePTFE membranes with xenograft (Todisco, 2010). Clinical VBA studies using non-resorbable membranes consistently report between 4 and $6 \mathrm{~mm}$ of vertical bone gain (Cucchi et al., 2017; Fontana et al., 2015; Ronda et al., 2014; Urban et al., 2019).

Although using space-making frameworks, including meshes and GBR membranes, has fewer complications than distraction osteogenesis or block grafting, flap dehiscence is a chief concern. Absorbable membranes have been associated with more complications than non-resorbable membranes (Lim et al., 2018). This contrast hold trues when VBA-only procedures are consideredfor these surgeries, absorbable membranes have complication rates of $23 \%$, whereas PTFE membranes have rates of $7 \%$ (Urban et al., 2019). However, not all non-resorbable membranes behave similarly. ePTFE membranes present with twice the number of the complications that dPTFE barriers do (Urban et al., 2019). Our study employing a novel PTFE mesh had a fairly low complication rate (3\%). Two complications involved membrane exposure at 1 or
8 weeks post-VBA, which agreed with a previous study reporting early and late exposure (as early as 1 week and up to 6 months postoperatively) (Lim et al., 2018).

\section{2 | Distinctive findings}

Most studies investigating VBA report only mean vertical bone gain (absolute bone gain) and fail to calculate the relative percentage of bone gain with respect to initial defect size. We detected significant indirect correlations between baseline vertical deficiency and amount of bone gained: as the baseline deficiency increased millimeter by millimeter, the probability of having complete regeneration decreased by 2.5 times. VBA achieved $6 \%-12 \%$ greater relative bone gain when used for smaller height deficiencies $(<5 \mathrm{~mm})$ than larger ones ( $\geq 5 \mathrm{~mm}$ ) (Figure $3 \mathrm{~b}$ ).

Ours may be the first VBA study to investigate the effect of defect location on height gain. We did not find significant differences between the maxilla and mandible in terms of bone gain. However, the location of the defect within either jaw statistically influenced the amount of augmentation generated. In the maxilla, absolute bone gain was statistically greater in the posterior than the anterior area, with a mean difference of $0.36 \mathrm{~mm}$. In contrast, the absolute 
bone gain in the mandible was statistically greater in the anterior than the posterior area, with a mean difference of $0.32 \mathrm{~mm}$. The mean height gain differences were $<0.5 \mathrm{~mm}$, which may have no clinical significance.

\subsection{Limitations and recommendations for future research}

As our study was a retrospective case series introducing a novel space-making mesh, rigorous comparisons to other studies were not viable. We postulate that the mesh macropores would enhance graft vascularization and therefore improve bone regeneration. Future studies should verify if this assumption is accurate via acquiring bone cores at re-entry to confirm regeneration/bone viability.

Surgeries were performed by a highly experienced clinician, and our outcomes may not be applicable to less seasoned practitioners. A prospective clinical trial is needed to compare vertical bone augmentation results using various space-making frameworks, such as PTFE mesh and ePTFE, dPTFE. Future comparison should be performed particularly for newer space-maintaining devices such as custom titanium mesh and custom-made block grafts. Using a larger patient sample with more even distribution would verify or refute our findings regarding baseline vertical deficiencies, defect location, patient factors, complications, and bone gain. Such analyses will help in case preparation and materials selection. Finally, data relative to peri-implant bone levels and survival rate after GBR procedure was not assessed in the present study. A prospective study will be performed to explore the long-term performance of implants placed in vertically augmented bone.

\section{5 | CONCLUSIONS}

As the group 4 of the 15th European Workshop on Periodontology on Bone Regeneration concluded, vertical ridge augmentation is a highly technique sensitive surgical intervention to be performed only by highly experienced clinicians (Jepsen et al., 2019).

1. Vertical ridge augmentation with titanium-reinforced PTFE mesh and a mixture of autologous bone and xenograft may be a safe and predictable procedure.

2. The extent of the baseline vertical deficiency influences the percentage of bone augmentation achieved, with each additional millimeter increasing the likelihood of incomplete bone regeneration by 2.5 times.

3. The defect location may exert a small influence on the amount of bone gained $(<0.5 \mathrm{~mm})$.

\section{ACKNOWLEDGEMENTS}

Dr. Urban received honorarium for speaking for Osteogenics Biomedical Company and Geistlich Pharma. Patent pending for perforated PTFE. Dr. Wang received honorarium for speaking for Osteogenics Biomedical Company. The rest coauthors do not have any financial interests neither directly nor indirectly in the companies whose materials were evaluated in this study.

\section{AUTHOR CONTRIBUTIONS}

Andras Forster: Data curation (supporting); Investigation (supporting); Validation (supporting). Zoltan Barath: Conceptualization (supporting); Investigation (supporting); Methodology (lead); Resources (supporting); Supervision (supporting); Writing-review \& editing (supporting).

\section{DATA AVAILABILITY STATEMENT}

Data available upon request from the authors.

\section{ORCID}

Istvan A. Urban (iD https://orcid.org/0000-0002-9368-4047

Andrea Ravidà iD https://orcid.org/0000-0002-3029-8130

Hom-Lay Wang iD https://orcid.org/0000-0003-4238-1799

\section{REFERENCES}

Buser, D., Chappuis, V., Belser, U. C., \& Chen, S. (2017). Implant placement post extraction in esthetic single tooth sites: When immediate, when early, when late? Periodontology 2000, 73(1), 84-102. https:// doi.org/10.1111/prd.12170

Canullo, L., \& Sisti, A. (2010). Early implant loading after vertical ridge augmentation (VRA) using e-PTFE titanium-reinforced membrane and nano-structured hydroxyapatite: 2-year prospective study. European Journal of Oral Implantology, 3(1), 59-69.

Chiapasco, M., \& Casentini, P. (2018). Horizontal bone-augmentation procedures in implant dentistry: Prosthetically guided regeneration. Periodontology 2000, 77(1), 213-240. https://doi.org/10.1111/ prd.12219

Chiapasco, M., \& Zaniboni, M. (2009). Clinical outcomes of GBR procedures to correct peri-implant dehiscences and fenestrations: A systematic review. Clinical Oral Implants Research, 20(Suppl 4), 113-123. https://doi.org/10.1111/j.1600-0501.2009.01781.x

Cordaro, L., Amade, D. S., \& Cordaro, M. (2002). Clinical results of alveolar ridge augmentation with mandibular block bone grafts in partially edentulous patients prior to implant placement. Clinical Oral Implants Research, 13(1), 103-111. https://doi. org/10.1034/j.1600-0501.2002.130113.x

Cucchi, A., Vignudelli, E., Napolitano, A., Marchetti, C., \& Corinaldesi, G. (2017). Evaluation of complication rates and vertical bone gain after guided bone regeneration with non-resorbable membranes versus titanium meshes and resorbable membranes. A randomized clinical trial. Clinical Implant Dentistry and Related Research, 19(5), 821-832. https://doi.org/10.1111/cid.12520

Elnayef, B., Porta, C., Suarez-Lopez Del Amo, F., Mordini, L., GargalloAlbiol, J., \& Hernandez-Alfaro, F. (2018). The fate of lateral ridge augmentation: A systematic review and meta-analysis. International Journal of Oral and Maxillofacial Implants, 33(3), 622-635. https://doi. org/10.11607/jomi.6290

Fontana, F., Grossi, G. B., Fimano, M., \& Maiorana, C. (2015). Osseointegrated implants in vertical ridge augmentation with a nonresorbable membrane: A retrospective study of 75 implants with 1 to 6 years of follow-up. International Journal of Periodontics and Restorative Dentistry, 35(1), 29-39. https://doi.org/10.11607/prd.2136

Hammerle, C. H. F., \& Tarnow, D. (2018). The etiology of hard- and softtissue deficiencies at dental implants: A narrative review. Journal of 
Periodontology, 89(Suppl 1), S291-S303. https://doi.org/10.1002/ JPER.16-0810

Jepsen, S., Schwarz, F., Cordaro, L., Derks, J., Hammerle, C. H. F., HeitzMayfield, L. J., Hernández-Alfaro, F., Meijer, H. J. A., Naenni, N., OrtizVigón, A., Pjetursson, B., Raghoebar, G. M., Renvert, S., Rocchietta, I., Roccuzzo, M., Sanz-Sánchez, I., Simion, M., Tomasi, C., Trombelli, L., \& Urban, I. (2019). Regeneration of alveolar ridge defects. Consensus report of group 4 of the 15th European Workshop on Periodontology on Bone Regeneration. Journal of Clinical Periodontology, 46 (Suppl 21), 277-286. https://doi.org/10.1111/jcpe.13121

Jung, R. E., Fenner, N., Hammerle, C. H., \& Zitzmann, N. U. (2013). Longterm outcome of implants placed with guided bone regeneration (GBR) using resorbable and non-resorbable membranes after 12-14 years. Clinical Oral Implants Research, 24(10), 1065-1073. https://doi. org/10.1111/j.1600-0501.2012.02522.x

Lim, G., Lin, G. H., Monje, A., Chan, H. L., \& Wang, H. L. (2018). Wound healing complications following guided bone regeneration for ridge augmentation: A systematic review and meta-analysis. International Journal of Oral and Maxillofacial Implants, 33(1), 41-50. https://doi. org/10.11607/jomi.5581

Machtei, E. E. (2001). The effect of membrane exposure on the outcome of regenerative procedures in humans: A meta-analysis. Journal of Periodontology, 72(4), 512-516. https://doi.org/10.1902/ jop.2001.72.4.512

Mailoa, J., Miron, R. J., \& Wang, H. L. (2017). Risk indicators and prevention of implant soft-tissue complications: Interproximal papillae loss and midfacial implant mucosal recessions. Compendium of Continuing Education in Dentistry, 38(7), 436-443, quiz 444.

Maiorana, C., Beretta, M., Salina, S., \& Santoro, F. (2005). Reduction of autogenous bone graft resorption by means of bio-oss coverage: $A$ prospective study. International Journal of Periodontics \& Restorative Dentistry, 25(1), 19-25.

McGinnis, M., Larsen, P., Miloro, M., \& Beck, F. M. (1998). Comparison of resorbable and nonresorbable guided bone regeneration materials: A preliminary study. International Journal of Oral and Maxillofacial Implants, 13(1), 30-35.

Melcher, A. H. (1976). On the repair potential of periodontal tissues. Journal of Periodontology, 47(5), 256-260. https://doi.org/10.1902/ jop.1976.47.5.256

Milinkovic, I., \& Cordaro, L. (2014). Are there specific indications for the different alveolar bone augmentation procedures for implant placement? A systematic review. International Journal of Oral and Maxillofacial Surgery, 43(5), 606-625. https://doi.org/10.1016/j. ijom.2013.12.004

Nowzari, H., \& Slots, J. (1995). Microbiologic and clinical study of polytetrafluoroethylene membranes for guided bone regeneration around implants. International Journal of Oral and Maxillofacial Implants, 10(1), 67-73.

Papageorgiou, S. N., Papageorgiou, P. N., Deschner, J., \& Gotz, W. (2016). Comparative effectiveness of natural and synthetic bone grafts in oral and maxillofacial surgery prior to insertion of dental implants: Systematic review and network meta-analysis of parallel and cluster randomized controlled trials. Journal of Dentistry, 48, 1-8. https://doi. org/10.1016/j.jdent.2016.03.010

Park, S. H., Brooks, S. L., Oh, T. J., \& Wang, H. L. (2009). Effect of ridge morphology on guided bone regeneration outcome: Conventional tomographic study. Journal of Periodontology, 80(8), 1231-1236. https://doi.org/10.1902/jop.2009.090090

Polimeni, G., Xiropaidis, A. V., \& Wikesjo, U. M. (2006). Biology and principles of periodontal wound healing/regeneration. Periodontology 2000, 41(1), 30-47. https://doi.org/10.1111/j.1600-0757.2006.00157.x

Ricci, L., Perrotti, V., Ravera, L., Scarano, A., Piattelli, A., \& lezzi, G. (2013). Rehabilitation of deficient alveolar ridges using titanium grids before and simultaneously with implant placement: A systematic review. Journal of Periodontology, 84(9), 1234-1242. https://doi. org/10.1902/jop.2012.120314

Roccuzzo, M., Savoini, M., Dalmasso, P., \& Ramieri, G. (2017). Long-term outcomes of implants placed after vertical alveolar ridge augmentation in partially edentulous patients: A 10 -year prospective clinical study. Clinical Oral Implants Research, 28(10), 1204-1210. https://doi. org/10.1111/clr.12941

Ronda, M., Rebaudi, A., Torelli, L., \& Stacchi, C. (2014). Expanded vs. dense polytetrafluoroethylene membranes in vertical ridge augmentation around dental implants: A prospective randomized controlled clinical trial. Clinical Oral Implants Research, 25(7), 859-866. https:// doi.org/10.1111/clr.12157

Rothamel, D., Schwarz, F., Sculean, A., Herten, M., Scherbaum, W., \& Becker, J. (2004). Biocompatibility of various collagen membranes in cultures of human PDL fibroblasts and human osteoblast-like cells. Clinical Oral Implants Research, 15(4), 443-449. https://doi. org/10.1111/j.1600-0501.2004.01039.x

Sbordone, L., Toti, P., Menchini-Fabris, G. B., Sbordone, C., Piombino, P., \& Guidetti, F. (2009). Volume changes of autogenous bone grafts after alveolar ridge augmentation of atrophic maxillae and mandibles. International Journal of Oral and Maxillofacial Surgery, 38(10), 1059-1065. https://doi.org/10.1016/j.ijom.2009.06.024

Silness, J., \& Loe, H. (1964). Periodontal disease in pregnancy. II. Correlation between oral hygiene and periodontal condition. Acta Odontologica Scandinavica, 22, 121-135. https://doi. org/10.3109/00016356408993968

Todisco, M. (2010). Early loading of implants in vertically augmented bone with non-resorbable membranes and deproteinised anorganic bovine bone. An uncontrolled prospective cohort study. European Journal of Oral Implantology, 3(1), 47-58.

Urban, I. A., Jovanovic, S. A., \& Lozada, J. L. (2009). Vertical ridge augmentation using guided bone regeneration (GBR) in three clinical scenarios prior to implant placement: A retrospective study of 35 patients 12 to 72 months after loading. International Journal of Oral and Maxillofacial Implants, 24(3), 502-510.

Urban, I. A., Lozada, J. L., Jovanovic, S. A., Nagursky, H., \& Nagy, K. (2014). Vertical ridge augmentation with titanium-reinforced, dense-PTFE membranes and a combination of particulated autogenous bone and anorganic bovine bone-derived mineral: A prospective case series in 19 patients. International Journal of Oral and Maxillofacial Implants, 29(1), 185-193. https://doi.org/10.11607/jomi.3346

Urban, I. A., Monje, A., Nevins, M., Nevins, M. L., Lozada, J. L., \& Wang, H. L. (2016). Surgical management of significant maxillary anterior vertical ridge defects. International Journal of Periodontics \& Restorative Dentistry, 36(3), 329-337. https://doi.org/10.11607/ prd.2644

Urban, I. A., Monje, A., \& Wang, H. L. (2015). Vertical ridge augmentation and soft tissue reconstruction of the anterior atrophic maxillae: A case series. International Journal of Periodontics \& Restorative Dentistry, 35(5), 613-623. https://doi.org/10.11607/prd.2481

Urban, I. A., Monje, A., Wang, H. L., Lozada, J., Gerber, G., \& Baksa, G. (2017). Mandibular regional anatomical landmarks and clinical implications for ridge augmentation. International Journal of Periodontics \& Restorative Dentistry, 37(3), 347-353. https://doi.org/10.11607/ prd.3199

Urban, I. A., Montero, E., Monje, A., \& Sanz-Sanchez, I. (2019). Effectiveness of vertical ridge augmentation interventions: A systematic review and meta-analysis. Journal of Clinical Periodontology, 46(Suppl 21), 319-339. https://doi.org/10.1111/jcpe.13061

Wang, H. L., \& Boyapati, L. (2006). "PASS" principles for predictable bone regeneration. Implant Dentistry, 15(1), 8-17. https://doi. org/10.1097/01.id.0000204762.39826.0f

Wen, S. C., Fu, J. H., \& Wang, H. L. (2018). Effect of deproteinized bovine bone mineral at implant dehiscence defects grafted by the sandwich 
bone augmentation technique. International Journal of Periodontics \& Restorative Dentistry, 38(1), 79-85. https://doi.org/10.11607/ prd.2931

\section{SUPPORTING INFORMATION}

Additional supporting information may be found online in the Supporting Information section.
How to cite this article: Urban IA, Saleh MHA, Ravidà A, Forster A, Wang H-L, Barath Z. Vertical bone augmentation utilizing a titanium-reinforced PTFE mesh: A multi-variate analysis of influencing factors. Clin Oral Impl Res. 2021;00:112. https://doi.org/10.1111/clr.13755 